\title{
Hadron spectroscopy at BESIII
}

\author{
Xiaolin Kang*t \\ Institute of High Energy Physics, Chinese Academy of Science \\ E-mail: kangxl@ihep.ac.cn
}

BESIII has many results on the hadron spectroscopy in the tau-charm energy region. In this proceeding, I will review the current status of the exotic $Z_{c}$ states, $X$ (3823), $X(3872)$, several $X$ states around 1.8-1.9 GeV/c $c^{2}$, the partial wave analysis of $J / \psi \rightarrow \gamma \eta \eta$, and the baryon spectroscopy in the processes $\psi(3686) \rightarrow K^{-} \Lambda \bar{\Xi}^{+}$and $\psi(3686) \rightarrow p \bar{p} \pi^{0}$.

XXVII International Symposium on Lepton Photon Interactions at High Energies 17-22 August 2015

Ljubljana, Slovenia

* Speaker.

$\dagger$ on behalf of BESIII Collaboration 


\section{Current status of the exotic $Z_{c}$ states}

The $Z_{c}(3900)^{ \pm}$was first observed by BESIII and Belle collaboration in 2013 [1, 2], then confirmed by CLEOc data [3]. The $Z_{c}(3900)^{ \pm}$couples to charmonium and has an electric charge, it can not be a conventional $c \bar{c}$ state. Proposed interpretations for $Z_{c}^{ \pm}$include hadronic molecules, hadroquarkonia, tetra-quark states, kinematic effects and so on. Subsequently, based on the data sets accumulated at center-of-mass above $4 \mathrm{GeV}$, a large amount of charged charmonium-like states, $Z_{c}(3885)^{ \pm}, Z_{c}(4020)^{ \pm}, Z_{c}(4025)^{ \pm}$, as well as their isospin partners, the neutral states $Z_{c}(3900)^{0}$, $Z_{c}(3885)^{0}, Z_{c}(4020)^{0}, Z_{c}(4025)^{0}$, were observed $[4,5,6,7,8,9,10]$, which are displayed in Fig. 1. The masses, widths and decay modes for $Z_{c}$ states are summarized in Table 1.

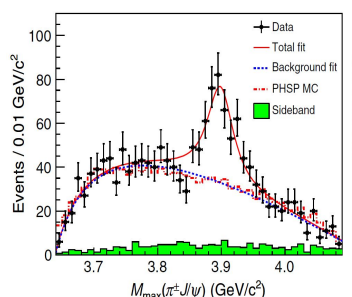

(a) $Z_{c}(3900)^{ \pm}$

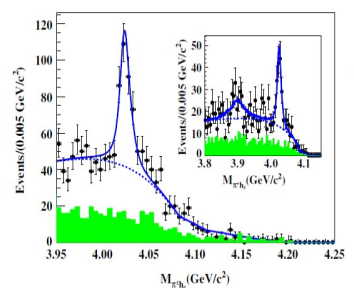

(e) $Z_{c}(4020)^{ \pm}$

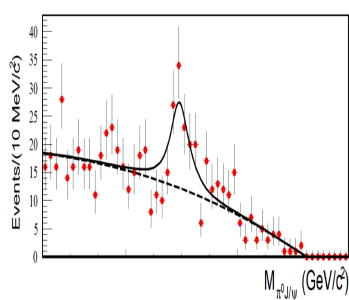

(b) $Z_{c}(3900)^{0}$

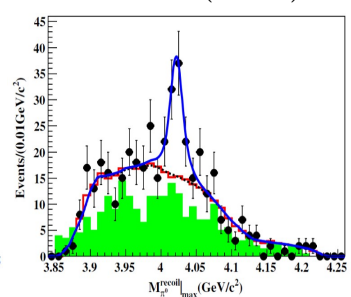

(f) $Z_{c}(4020)^{0}$

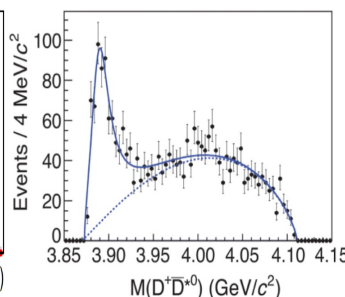

(c) $Z_{c}(3885)^{+}$

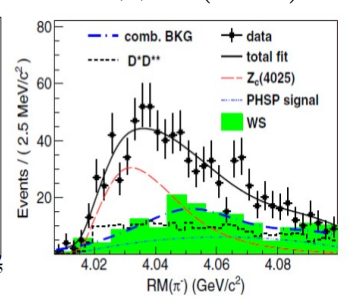

(g) $Z_{c}(4025)^{ \pm}$

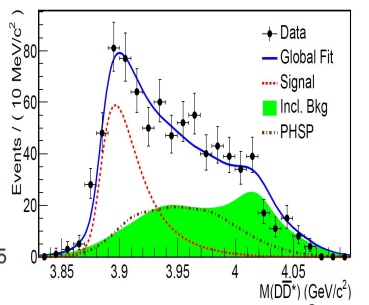

(d) $Z_{c}(3885)^{0}$

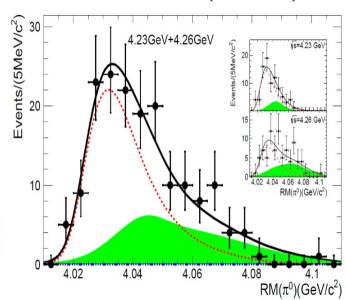

(h) $Z_{c}(4025)^{0}$

Figure 1: The observed $Z_{c}$ states at BESIII.

Table 1: The parameters and decay mode of the current $Z_{c}$ states at BESIII.

\begin{tabular}{lllll}
\hline \hline State & $\mathrm{M}\left(\mathrm{MeV} / \mathrm{c}^{2}\right)$ & $\Gamma(\mathrm{MeV})$ & Process & Decay \\
\hline$Z_{c}(3900)^{ \pm}$ & $3899.0 \pm 3.6 \pm 4.9$ & $46 \pm 10 \pm 20$ & $e^{+} e^{-} \rightarrow \pi^{+} \pi^{-} J \psi$ & $\pi^{ \pm} J / \psi$ \\
$Z_{c}(3900)^{0}$ & $3894.8 \pm 2.3 \pm 2.7$ & $29.6 \pm 8.2 \pm 8.2$ & $e^{+} e^{-} \rightarrow \pi^{0} \pi^{0} J \psi$ & $\pi^{0} J / \psi$ \\
$Z_{c}(3885)^{ \pm}$ & $3884.3 \pm 1.2 \pm 1.5$ & $24.8 \pm 2.1 \pm 2.6$ & $e^{+} e^{-} \rightarrow \pi^{ \pm}\left(D \bar{D}^{*}\right)^{\mp}$ & $\left(D \bar{D}^{*}\right)^{ \pm}$ \\
$Z_{c}(3885)^{0}$ & $3885.7_{-5.7}^{+4.3} \pm 8.4$ & $35_{-12}^{+11} \pm 15$ & $e^{+} e^{-} \rightarrow \pi^{0}\left(D \bar{D}^{*}\right)^{0}$ & $\left(D \bar{D}^{*}\right)^{0}$ \\
$Z_{c}(4020)^{ \pm}$ & $4022.9 \pm 0.8 \pm 2.7$ & $7.9 \pm 2.7 \pm 2.6$ & $e^{+} e^{-} \rightarrow \pi^{+} \pi^{-} h_{c}$ & $\pi^{ \pm} h_{c}$ \\
$Z_{c}(4020)^{0}$ & $4023.9 \pm 2.2 \pm 3.8$ & $7.9($ fixed $)$ & $e^{+} e^{-} \rightarrow \pi^{0} \pi^{0} h_{c}$ & $\pi^{0} h_{c}$ \\
$Z_{c}(4025)^{ \pm}$ & $4026.3 \pm 2.6 \pm 3.7$ & $24.8 \pm 5.6 \pm 7.7$ & $e^{+} e^{-} \rightarrow \pi^{ \pm}\left(D^{*} \bar{D}^{*}\right)^{\mp}$ & $\left(D^{*} \bar{D}^{*}\right)^{ \pm}$ \\
$Z_{c}(4025)^{0}$ & $4025.5_{-4.7}^{+2.0} \pm 3.1$ & $23.0 \pm 6.0 \pm 1.0$ & $e^{+} e^{-} \rightarrow \pi^{0}\left(D^{*} \bar{D}^{*}\right)^{0}$ & $\left(D^{*} \bar{D}^{*}\right)^{0}$ \\
\hline \hline
\end{tabular}

\section{2. $X(3823)$ and $X(3872)$ states}

In 2013, the Belle collaboration observed a new resonance $X(3823) \rightarrow \gamma \chi_{c 1}$ with $3.1 \sigma$ significance via the process $B \rightarrow K \gamma \chi_{c 1}$ [11]. Recently, the BESIII collaboration firmly established the 
$X(3823)$ resonance in the process $e^{+} e^{-} \rightarrow \pi^{+} \pi^{-} \gamma \chi_{c 1}$ with $4.67 f b^{-1}$ data set above $4 \mathrm{GeV}$ with $6.2 \sigma$ statistical significance [12], as shown in Fig. 2 (a). The mass is measured to be $3821.7 \pm 1.3 \pm$ $0.7 \mathrm{MeV} / c^{2}$ and its width is quite narrow, which agrees quite well with Belle. By further studying the properties of $X(3823)$, both BESIII and Belle support it's a perfect candidate for the missing $\psi\left(1^{3} D_{2}\right)$ charmonium state.

The $X(3872)$ is still the most puzzling particle more than 10 years after its discovery. In order to understand its true nature, various studies toward its properties are going. Among these efforts, the BESIII collaboration's recent search for $e^{+} e^{-} \rightarrow \gamma X$ (3872) process above $4 \mathrm{GeV}$ [13] looks quite interesting. BESIII has observed the $X(3872)$ state with $6.3 \sigma$ statistical significance, and measured its mass to be $3871.9 \pm 0.7 \pm 0.2 \mathrm{MeV} / c^{2}$, which agrees quite well with other experiments. BESIII also observed the decay $Y(4260) \rightarrow \gamma X(3872)$ for the first time, shown in Fig. 2 (b), and the radiative transition may hint a common nature for their internal structure.
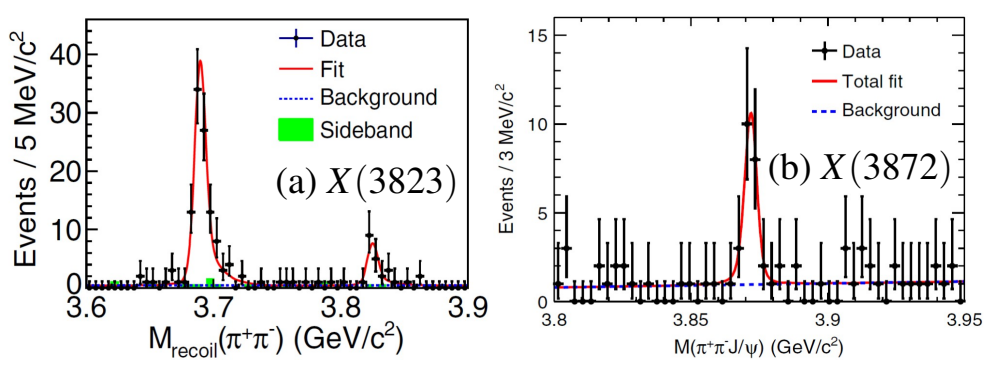

Figure 2: (a) $X(3823)$ and (b) the exotic $X(3872)$ state at BESIII.

\section{Light meson/baryon spectroscopy}

\section{1 $X$ states at the 1.8-1.9 $\mathrm{GeV} / c^{2}$ region}

Since the first observation of $X(1835)$ in $J / \psi \rightarrow \gamma \pi^{+} \pi^{-} \eta^{\prime}$ by the BESII experiment [14], possible interpretations including $p \bar{p}$ bound state, second radial excitation of the $\eta^{\prime}$, and pseudoscalar glueball have been proposed. Recently, a new process $J / \psi \rightarrow \gamma X(1835) \rightarrow \gamma K_{s}^{0} K_{s}^{0} \eta$ at low $K_{s}^{0} K_{s}^{0}$ mass was observed [15], shown in Fig. 3 (a), where the $K_{s}^{0} K_{s}^{0}$ system is dominanted by resonant $f_{0}(980)$ contribution. By performing partial wave analysis (PWA), the spin-parity is determined to be $J^{P C}=0^{-+}$. The mass and width are determined as $1844 \pm 9$ (stat) ${ }_{-25}^{+16}$ (syst) $\mathrm{MeV} / \mathrm{c}^{2}$ and $192_{-25}^{+16}(\text { stat })_{-43}^{+62}$ (syst) $\mathrm{MeV}$, respectively.

BESIII reported the first observation of the $X(1870)$ in $J / \psi \rightarrow \omega \pi^{+} \pi^{-} \eta$ in 2011 [16]. More recently, the spin-parity of the threshold structures, $X(p \bar{p})$, is determined as $0^{-+}$by the PWA of $J / \psi \rightarrow \gamma p \bar{p}$ at BESIII [17]. Its peak mass is determined to be $M=1832_{-5}^{+19}(\text { stat })_{17}^{+18}($ syst $)$ $\mathrm{MeV} / \mathrm{c}^{2}$, and its total width $\Gamma<76 \mathrm{MeV}$ at $90 \%$ C.L. While the spin-parity of the enhancement near $\omega \phi$ threshold, $X(1810)$, is confirmed to be $0^{++}$in the PWA of $J / \psi \rightarrow \gamma \omega \phi$ [18]. In 2013, a new structure at $1.84 \mathrm{GeV} / c^{2}, X(1840)$, is first observed in $J / \psi \rightarrow \gamma 3\left(\pi^{+} \pi^{-}\right)$[19]. The mass and width are measured to be $M=1842.2 \pm 4.2_{2.6}^{+7.1} \mathrm{MeV} / c^{2}$ and $\Gamma=83 \pm 14 \pm 11 \mathrm{MeV}$, where the mass is in agreement with those of $X(1835)$ and $X(p \bar{p})$, while its width is significantly different from either of them. The comparison of the masses and widths of the $X$ states at $1.8-1.9 \mathrm{GeV} / c^{2}$ mass region are displayed in Fig. 3 (b). Further studies are needed to establish the relationship between 
different experimental observations in this mass region and determine the nature of the underlying structures.

\subsection{PWA of $J \psi \rightarrow \gamma \eta \eta$}

Radiative $J / \psi$ decay is a gluon-rich process and has long been regarded as one of the most promising hunting ground for glueballs. A full PWA has been carried out for the selected $J / \psi \rightarrow$ $\gamma \eta \eta$ events using the relativistic covariant tensor amplitude method [20]. The fit indicates that the scalar resonances $f_{0}(1500), f_{0}(1710), f_{0}(2100)$ and the tensor resonances $f_{2}^{\prime}(1525), f_{2}(1810), f_{2}(2340)$ are needed in the process, as shown in Fig. 3 (a).

\subsection{Observation of two hyperons in $\psi(3686) \rightarrow K^{-} \Lambda \bar{\Xi}^{+}$}

In the decay $\psi(3686) \rightarrow K^{-} \Lambda \bar{\Xi}^{+}$, two hypersons $\Xi(1690)^{-}$and $\Xi(1820)^{-}$are observed with significance of $4.9 \sigma$ and $6.2 \sigma$, respectively [21]. Fig. 3 (b) shows the result of an unbinned maximum likelihood fit to $K^{-} \Lambda$ invariant mass spectrum. The fitted resonance parameters are consistent with the values in particle data group (PDG) within one standard deviation. This is the first time that $\Xi(1690)^{-}$and $\Xi(1820)^{-}$hyperons have been observed in charmonium decays, which would provide new information on charmonium decays to hyperons.
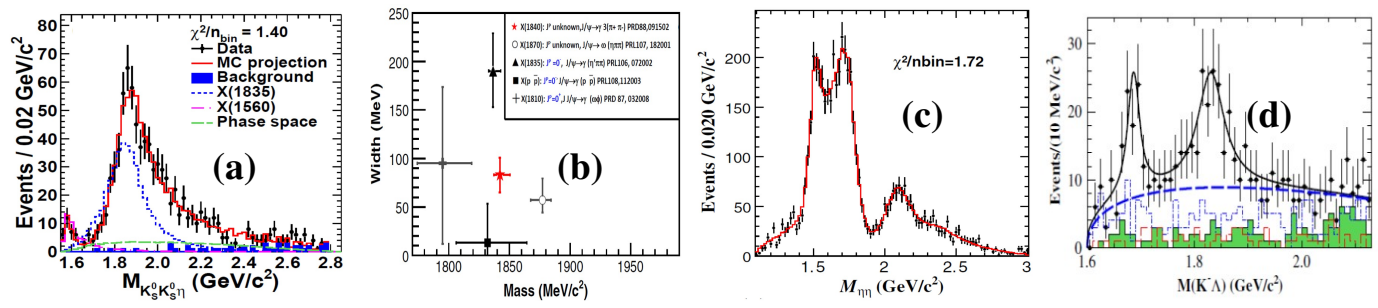

Figure 3: (a) The $X(1835)$ in $J / \psi \rightarrow \gamma X(1835) \rightarrow \gamma K_{s}^{0} K_{s}^{0} \eta$; (b) The comparison of the $X$ states at 1.8-1.9 $\mathrm{GeV} / c^{2}$ mass region; (c) The invariant mass spectrum of $\eta \eta$ in the $J / \psi \rightarrow \gamma \eta \eta$ and PWA fit projection; (d) The fit result of $K^{-} \Lambda$ invariant mass spectrum.

\subsection{Observation of two new $N^{*}$ resonances in $\psi(3686) \rightarrow p \bar{p} \pi^{0}$}

The decay of $\psi(3686) \rightarrow p \bar{p} \pi^{0}$ is thought to be dominated by two-body decays involving $N^{*}, \bar{N}^{*}$ states and a hypothetical $p \bar{p}$ resonance. By including the resonances with significance larger than $5 \sigma$ and $N(940)$ (according to the framework of soft $\pi$ meson theory), $7 N^{*}$ intermediate resonances are needed to describe the data well [22]. Among these, two new resonances, $N(2300)$ and $N(2570)$ are significant, one $1 / 2^{+}$resonance with a mass of $2300_{-30-0}^{+40+109} \mathrm{MeV} / \mathrm{c}^{2}$ and width of $340_{-30-58}^{+30+110} \mathrm{MeV} / \mathrm{c}^{2}$, and one $5 / 2^{-}$resonance with a mass of $2570_{-10-10}^{+19+34} \mathrm{MeV} / \mathrm{c}^{2}$ and width of $250_{-24-21}^{+14+69} \mathrm{MeV} / c^{2}$. For the remaining $5 N^{*}$ intermediate resonances $[N(1440), N(1520), N(1535)$, $N(1650)$, and $N(1720)]$, the analysis yields mass and width values that are consistent with those from established resonances.

\section{Summary}

Based on the data sets collected with the BESIII detector, some significant progress in charmonium(-like) studies have been presented. To make clear the nature of some new 

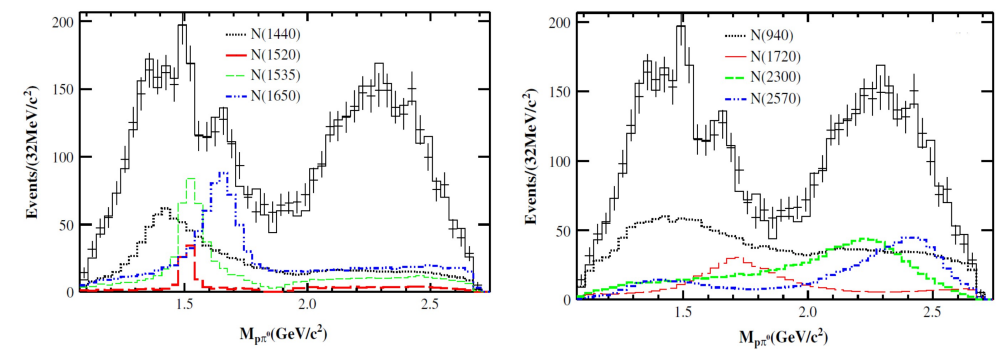

Figure 4: The PWA result of $\psi(3686) \rightarrow p \bar{p} \pi^{0}$.

observations, such as the quantum numbers of the exotic $Z_{c}$ states and the structures in 1.8-1.9 $\mathrm{GeV} / c^{2}$ region, more studies are needed. With some analysis are on going, we expect more results will come up soon.

\section{References}

[1] M. Ablikim, et al. (BESIII Collaboration), Phys. Rev. Lett. 110, 252001 (2013).

[2] Z. Q. Liu, et al. (Belle Collaboration), Phys. Rev. Lett. 110, 252002 (2013).

[3] T. Xiao, et al. Phys. Lett. B 727, 366 (2013).

[4] M. Ablikim, et al. (BESIII Collaboration), Phys. Rev. Lett. 112, 022001 (2014).

[5] M. Ablikim, et al. (BESIII Collaboration), Phys. Rev. Lett. 111, 242001 (2013).

[6] M. Ablikim, et al. (BESIII Collaboration), Phys. Rev. Lett. 112, 132001 (2014).

[7] M. Ablikim, et al. (BESIII Collaboration), Phys. Rev. Lett. 115, 112003 (2015).

[8] M. Ablikim, et al. (BESIII Collaboration), arXiv:1509.05620.

[9] M. Ablikim, et al. (BESIII Collaboration), Phys. Rev. Lett. 113, 212002 (2014).

[10] M. Ablikim, et al. (BESIII Collaboration), Phys. Rev. Lett. 110, 252001 (2013).

[11] V. Bhardwaj, et al. (Belle Collaboration), Phys. Rev. Lett. 111, 032001 (2013).

[12] M. Ablikim, et al. (BESIII Collaboration), Phys. Rev. Lett. 115, 011803 (2015).

[13] M. Ablikim, et al. (BESIII Collaboration), Phys. Rev. Lett. 112, 092001 (2014).

[14] M. Ablikim, et al. (BESII Collaboration), Phys. Rev. Lett. 95, 262001 (2005).

[15] M. Ablikim, et al. (BESIII Collaboration), Phys. Rev. Lett. 115, 091803 (2015).

[16] M. Ablikim, et al. (BESIII Collaboration), Phys. Rev. Lett. 107, 182001 (2011).

[17] M. Ablikim, et al. (BESIII Collaboration), Phys. Rev. Lett. 108, 112003 (2012).

[18] M. Ablikim, et al. (BESIII Collaboration), Phys. Rev. D 87, 032008 (2013).

[19] M. Ablikim, et al. (BESIII Collaboration), Phys. Rev. D 88, 091502 (2013).

[20] M. Ablikim, et al. (BESIII Collaboration), Phys. Rev. D 87, 092009 (2013).

[21] M. Ablikim, et al. (BESIII Collaboration), Phys. Rev. D 91, 092006 (2015).

[22] M. Ablikim, et al. (BESIII Collaboration), Phys. Rev. Lett. 110, 022001 (2013). 